\title{
Synthesis, Characterization, and Antimicrobial Activity of CoO Nanoparticles from a Co (II) Complex Derived from Polyvinyl Alcohol and Aminobenzoic Acid Derivative
}

\author{
Maged S. Al-Fakeh $\mathbb{D D}^{1,2}$ and Roaa O. Alsaedi ${ }^{1}$ \\ ${ }^{1}$ Department of Chemistry, College of Science, Qassim University, Buraidah 51452, Saudi Arabia \\ ${ }^{2}$ Taiz University, Taiz, Yemen \\ Correspondence should be addressed to Maged S. Al-Fakeh; alfakehmaged@yahoo.com
}

Received 27 December 2020; Revised 6 April 2021; Accepted 19 April 2021; Published 27 April 2021

Academic Editor: Marcelino M. Oliveira

Copyright (C) 2021 Maged S. Al-Fakeh and Roaa O. Alsaedi. This is an open access article distributed under the Creative Commons Attribution License, which permits unrestricted use, distribution, and reproduction in any medium, provided the original work is properly cited.

\begin{abstract}
Cobalt oxide nanoparticles ( $\mathrm{CoO}$ NPs) were synthesized by the calcination method from the Co (II) complex which has the formula $\left[\mathrm{Co}(\mathrm{PVA})(\mathrm{P}-\mathrm{ABA})\left(\mathrm{H}_{2} \mathrm{O}\right)_{3}\right], \mathrm{PVA}=$ polyvinyl alcohol, and $P$-ABA = para-aminobenzoic acid. The calcination temperature was $550^{\circ} \mathrm{C}$, and the products were characterized by element analysis, thermal analyses (TGA and DTA), Fourier transform infrared spectroscopy (FTIR), X-ray diffraction (XRD), UV-Vis spectra, and scanning electron microscopy (SEM) techniques. The kinetic and thermodynamic parameters $\left(\Delta \mathrm{H}^{*}, \Delta \mathrm{G}^{*}\right.$, and $\left.\Delta \mathrm{S}^{*}\right)$ for the cobalt (II) complex are calculated. The charges been carried by the atoms cause dipole moment 10.53 and 3.84 debye and total energy $11.04 \times 10^{2}$ and $24.80 \times 10^{2} k \mathrm{Cal} \mathrm{mol}^{-1}$ for the Co (II) complex and cobalt oxide, respectively. X-ray diffraction confirmed that the resulting oxide was pure single-crystalline CoO nanoparticles. Scanning electron microscopy indicating that the crystallite size of cobalt oxide nanocrystals was in the range of 36-54 nm. Finally, the antimicrobial activity of cobalt oxide nanoparticles was evaluated using four bacterial strains and one fungal strain. Two strains of Gram-positive cocci (Staphylococcus aureus and Enterococcus faecalis), two strains of Gram-negative bacilli (Escherichia coli and Pseudomonas aeruginosa), and one strain of yeast such as fungi (Candida albicans) were used in this study.
\end{abstract}

\section{Introduction}

Recently, metal oxide nanoparticles (MONPs) have been playing a very marked role in many fields of physics, chemistry, and other material science $[1,2]$. The transition metals are able to shape a large variety of oxide complexes. Metal oxide nanoparticles (MONPs) exhibit special chemical and physical properties due to their fixed size and more density of the corner. The particle size is likely to be influenced by three important groups of basic properties in more material. The first one involves the structural properties, specifically the lattice symmetry and cell parameter [3-5]. Cobalt oxide nanoparticles have been considerably used in various applications; pigments, rechargeable batteries, catalysts, magnets, sensors, solar energy absorbers, electrochromic devices, and biological activity [6-11]. Usage of cobalt oxide NPs in these applications is based on the physical and chemical properties of this oxide such as crystallinity, composition, crystal size, morphology, and surface area [12]. The physicochemical properties of metal oxide nanoparticles can be planned by synthesis using different methods $[13,14]$. The different methods used for synthesis of metal oxides are shown in Figure 1.

The calcination method produced very fine, homogeneous crystalline, high-product-purity, and well-dispersed nanoparticles [15-17]. Polyvinyl alcohol is one of the more important biodegradable, hydrophilic, and semicrystalline polymers, with excellent physical, chemical, and thermal stability. Additionally, polyvinyl alcohol (PVA) is a nontoxic, ecofriendly vinyl polymer soluble in water and highly biocompatible [18-21]. These properties for PVA led to a wide range of applications in pharmaceutical, packaging, 


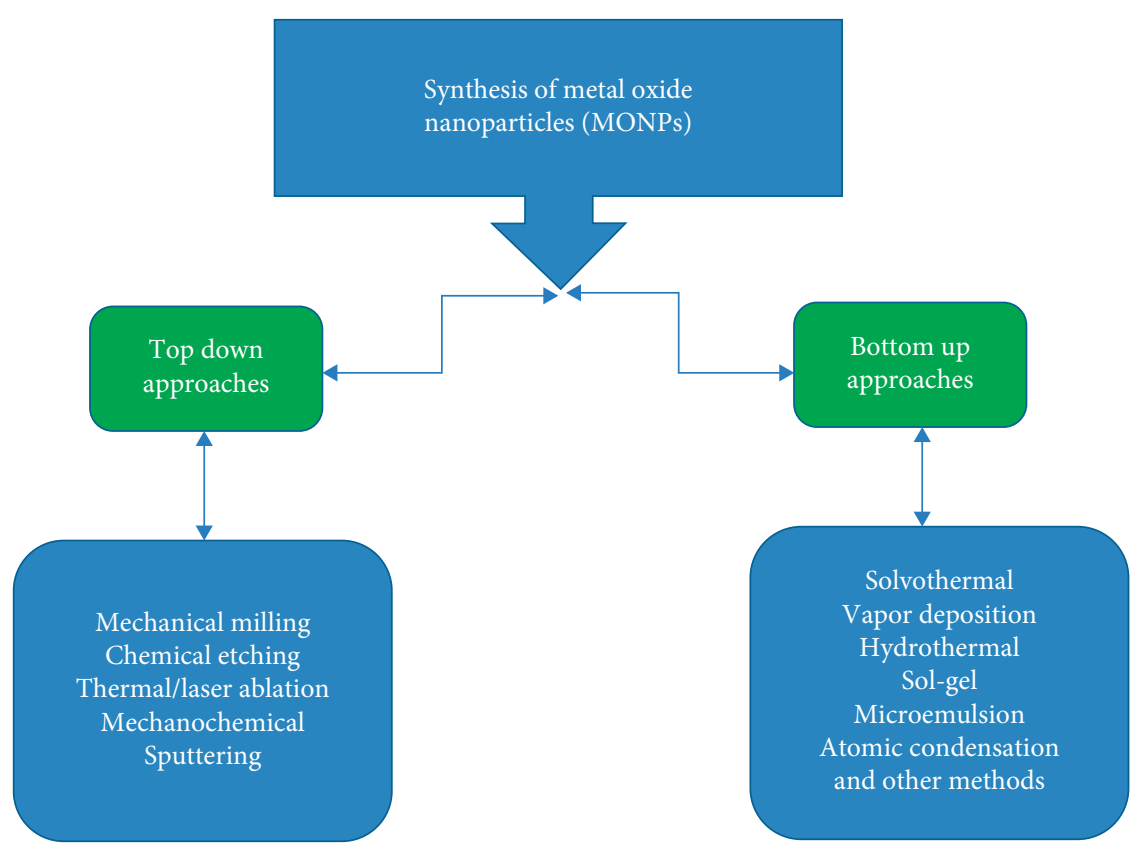

FIGURE 1: Physicochemical approaches of nanoparticle synthesis.

medical, cosmetic, and food industries [22]. There are groups that have investigated binary or mixed complexes between polyvinyl alcohol and metal ions [23, 24]. Metal compounds with 4-aminobenzoic acid are among the most explored types of complexes in coordination chemistry and play an important role in catalysis, other materials science, and biochemistry $[25,26]$. In this article, preparation and characterization of the cobalt (II) complex containing polyvinyl alcohol and $p$-aminobenzoic acid ligands are described and the resulting complex was used as a raw material for synthesis of nanoparticles of $\mathrm{CoO}$.

\section{Experimental}

2.1. Materials and Methods. Polyvinyl alcohol, paraaminobenzoic acid, and cobalt (II) chloride hexahydrates supplied by Sigma-Aldrich were used without further purification.

2.2. Preparation of $\left[\mathrm{Co}(\mathrm{PVA})(\mathrm{P}-\mathrm{ABA})\left(\mathrm{H}_{2} \mathrm{O}\right)_{3}\right]$. Polyvinyl alcohol ligand $(2 \mathrm{~g})$ was dissolved in $50 \mathrm{~mL}$ distilled water by stirring at $50^{\circ} \mathrm{C}$ for $60 \mathrm{~min}$; after cooling, a $\mathrm{CoCl}_{2} \cdot 6 \mathrm{H}_{2} \mathrm{O}$ solution $(6.42 \mathrm{~g}$ in $35 \mathrm{~mL}$ distilled water) was added drop wise to the polyvinyl alcohol solution under quickly stirring, and then, the $P$-aminobenzoic acid solution $(3.70 \mathrm{~g}$ in $30 \mathrm{~mL}$ ethanol) was added to the mixture. Finally, the mixture was refluxed for $3 \mathrm{~h}$ and then cooled. The light pink complex was separated by filtration, washed with $\mathrm{EtOH}$, and dried over $\mathrm{P}_{2} \mathrm{O}_{5}$. Anal. Calc. for $\mathrm{C}_{10} \mathrm{H}_{17} \mathrm{~N}_{2} \mathrm{CoO}_{7}$ : C, 35.73; H, 5.09; N, 8.33. Found: C, 34.96; H, 5.02; N, 8.14. Melting point: $295^{\circ} \mathrm{C}$. IR data: $v\left(\mathrm{~cm}^{-1}\right)=3420(\mathrm{~m}), 3292(\mathrm{~m}), 3152(\mathrm{~m}), 2922(\mathrm{~m})$, 2158 (m), 1598 (s), 1539 (s), $1454(\mathrm{~m}), 1395(\mathrm{~m}), 1084(\mathrm{~s})$, $880(\mathrm{~m}), 845(\mathrm{~m}), 782(\mathrm{~m}), 618(\mathrm{~m}), 590(\mathrm{~m})$, and $444(\mathrm{~m})$.

2.3. Preparation of Cobalt Oxide Nanoparticles by Calcinations. Direct calcination of the prepared
[Co(PVA) $\left.(P-A B A)\left(\mathrm{H}_{2} \mathrm{O}\right)_{3}\right]$ complex at $550^{\circ} \mathrm{C}$ with a calcination time of $3 \mathrm{~h}$ afforded $\mathrm{CoO}$ (NPs). The morphology and size of the as-prepared $\mathrm{CoO}$ sample was further investigated using scanning electron microscopy (SEM) and transmission electron microscopy (TEM).

2.4. Physical Measurements and Instrumentation. The $\mathrm{C}, \mathrm{H}$, and $\mathrm{N}$ contents of the solid Co (II) complex were determined by using an Elemental Analyser system GmbhVario El analyzer. FT-IR spectra of the compound and cobalt (II) oxide were obtained by the $\mathrm{KBr}$ disc technique; in the wavenumber range of $4000-400 \mathrm{~cm}^{-1}$, using a Thermo Nicolet (6700) FT-IR spectrophotometer. The electronic absorption spectral measurements in the ultraviolet and visible regions were carried out in dimethyl sulfoxide on a UV-2102 PC Shimadzu spectrophotometer using a $1 \mathrm{~cm}$ matched quartz cell in the wavelength range 200 to 900 nanometer. The magnetic susceptibility of the Co (II) complex was measured at room temperature using a magnetic susceptibility balance of type MSB-Auto. TGA and DTA analyses were completed employing a Shimadzu DTG60 instrument using a heating rate of $10^{\circ} \mathrm{C} / \mathrm{min}$ in the air atmosphere. The XRD patterns of the $\mathrm{CoO}$ NPs were registered using an XRD diffractometer Model (PW 1710) control unit (Philips). The anode material was copper (II) $\mathrm{K} \alpha$ $(\lambda=1.54180 \AA), 40 \mathrm{KV} 30 \mathrm{~mA}$; optics: automatic, divergence slit. The scanning electron microscope (SEM) used was JEOL JFC-1100E ION SPUTTERING, DEVICE, JEOL JSM (5400LV) SEM.

\subsubsection{Theoretical Calculations}

(1) Density Functional Theory (DFT). Density functional theory (DFT) calculations were accomplished on model 
complexes derived from the experimental structure data using the Amsterdam Density Functional (ADF) program [27].

\subsubsection{Antimicrobial Activity}

(1) Bacterial and Fungal Strains. The antimicrobial activity of cobalt oxide NPs was evaluated using four bacterial strains and one fungal strain. Two strains of Gram-positive cocci (Staphylococcus aureus and Enterococcus faecalis), two strains of Gam-negative bacilli (Escherichia coli, and Pseudomonas aeruginosa), and one stain of yeast such as fungi (Candida albicans) were used in this study.

(2) Inoculum and Cobalt (II) Oxide Nanoparticles Preparation. Bacterial and fungal inocula were prepared from fresh pure cultures in Muller Hinton broth. Each bacterial and fungal suspension were compared with $0.5 \mathrm{McF}$ arland standard. The $\mathrm{CoO}$ nanoparticles solution for antimicrobial activity was prepared by diluting powdered cobalt oxide nanoparticles with $1 \%$ DMSO.

(3) Agar Well Diffusion Assay. The antimicrobial activity of cobalt oxide nanoparticles against the selected microorganisms was evaluated using the agar well diffusion assay. The bacterial inoculum was spread on the Muller-Hinton agar using a sterile cotton swab by the lawn culture technique. After inoculation, we made 2 wells in the agar plate with the help of the backside blue micropipette tips. Then, cobalt oxide nanoparticle extracts $(100 \mu \mathrm{L})$ were added to one well and 1\% DMSO to another well.

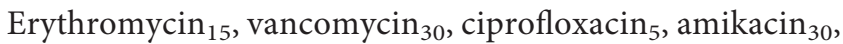
and ketoconazole ${ }_{50}$ were used as a positive control. The plates were then incubated for $18-24 \mathrm{hrs}$ at $37^{\circ} \mathrm{C}$. After incubation, the zone of inhibition around the wells was observed, and the zone diameter was measured in millimeter $(\mathrm{mm})$ by using a ruler.

\section{Results and Discussion}

The Co (II) complex was prepared by the reaction of polyvinyl alcohol and $p$-aminobenzoic acid in stoichiometric proportions to yield the identical compound according to Scheme 1.

This Co (II) compound was prepared and used as a precursor for cobalt oxide nanoparticles (CoO NPs) by the calcination method. The data correspond to the metal: L1: L2 ratio of the 1:1:1. Also, the complex is air stable, insoluble in common organic solvents, but partially soluble in dimethylsulphoxide (DMSO). The molar conductivity was determined in DMSO with value in the $45 \mathrm{ohm}^{-1} \mathrm{~cm}^{2} \mathrm{~mol}^{-1}$ for the Co (II) compound.

3.1. FT-IR Spectra. The FT-IR spectrum of the Co (II) complex prepared show an absorption peak was confirmed at $\nu\left(1178 \mathrm{~cm}^{-1}\right)$, and this band has been employed as an estimate polyvinyl alcohol structure because it is a semicrystalline synthetic polymer able to form domains depending on different approach parameters [28]. Additionally, the occurrence of hydrogen-bonded oxygenhydrogen stretching $[29,30]$ and antisymmetric stretching vibrations of both $\mathrm{CH}_{2}$ and $\mathrm{COC}$ at $2921 \mathrm{~cm}^{-1}$ and $1138 \mathrm{~cm}^{-1}$ for the Co (II) complex, respectively, is found. On the other hand, for polyvinyl alcohol ligand cross linked by the cobalt (II) compound, the band maximum corresponding to the bonded $\mathrm{OH}$ group at $3420 \mathrm{~cm}^{-1}$, was shifted to lower frequencies, $3292 \mathrm{~cm}^{-1}$. The wavenumbers of bands responsible for the amine group in the FT-IR of Co (II) also change in comparison to free $P$-aminobenzoic acid ligand. The $(P-\mathrm{ABA})$ ligand displaying a band at $1640 \mathrm{~cm}^{-1}$ for the imine $v(C=N)$ group, which results from the Schiff base condensation of $P$-ABA, was shifted to a lower frequency of $1626 \mathrm{~cm}^{-1}$ after complexation [29]. Finally, the appearance of bands at $548 \mathrm{~cm}^{-1}$ and $444 \mathrm{~cm}^{-1}$ corresponds to $v$ (metal$\mathrm{O})$ and $\nu$ (metal-N), respectively [31] (Figure 2).

3.2. Electronic Spectra and Magnetic Moments. The electronic spectra of the Co (II) complex and CoO NPs have been recorded in the DMSO solvent. The spectra show two distinct bands in the 32,258 and $26,455 \mathrm{~cm}^{-1}$ which corresponds to $\pi \longrightarrow \pi^{*}$ and $n \longrightarrow \pi^{*}$ transitions within polyvinyl alcohol and $P$-aminobenzoic acid moieties [32, 33], respectively. There are characteristic bands assigned to the $\mathrm{d}-\mathrm{d}$ transitions in the Co (II) complex typical of an octahedral structure (Figures 3 and 4). Additionally, the cobalt ((II) compound exhibits a d-d band in $19,230 \mathrm{~cm}^{-1}$, and the magnetic moments value was $4.36 \mathrm{BM}$ typical for the octahedral Co (II) complex [34]. The energy of the cobalt (II) complex elucidates that the difference in energy between high occupied molecular orbital (HOMO) and lower unoccupied molecular orbital (LUMO) is called the (HOMOLUMO) gap; also, this difference in energy between these two boundary orbitals indicated to form the strength and stability of the cobalt (II) complex. DFT MOs in their HOMO-LUMO zone for compounds are displayed in Figures 5 and 6.

3.3. Thermal Analysis. The TG, DTG, and DTA curves (Figure 7) of the Co (II) complex show that the thermal decomposition stages of the compound involve four stages. The four steps occur in the temperature ranges 42-145, $147-205,207-310$, and $312-600^{\circ} \mathrm{C}$. The first mass loss correlates well with the release of the three $\mathrm{H}_{2} \mathrm{O}$ molecules (calc. $16.09 \%$, found: $15.92 \%$ ) with a corresponding DTG peak at $120^{\circ} \mathrm{C}$ accompanied with a broad endothermic peak in the D.T.A curve at $122^{\circ} \mathrm{C}$. The second, third, and fourth steps related to decomposition of the rest of the organic ligands. These steps are marked on the DTG curve at 158, 250, and $548^{\circ} \mathrm{C}$ with correlating exothermic peaks in the D.T.A curve at 160,252 , and $550^{\circ} \mathrm{C}$, respectively. The residue corresponds to a cobalt oxide (calc. $22.29 \%$, found: $20.84 \%$ ).

3.3.1. Kinetic Analysis and Thermodynamic Parameters for the Co (II) Complex. Nonisothermal kinetic studies of the Co (II) complex were accomplished applying two different 


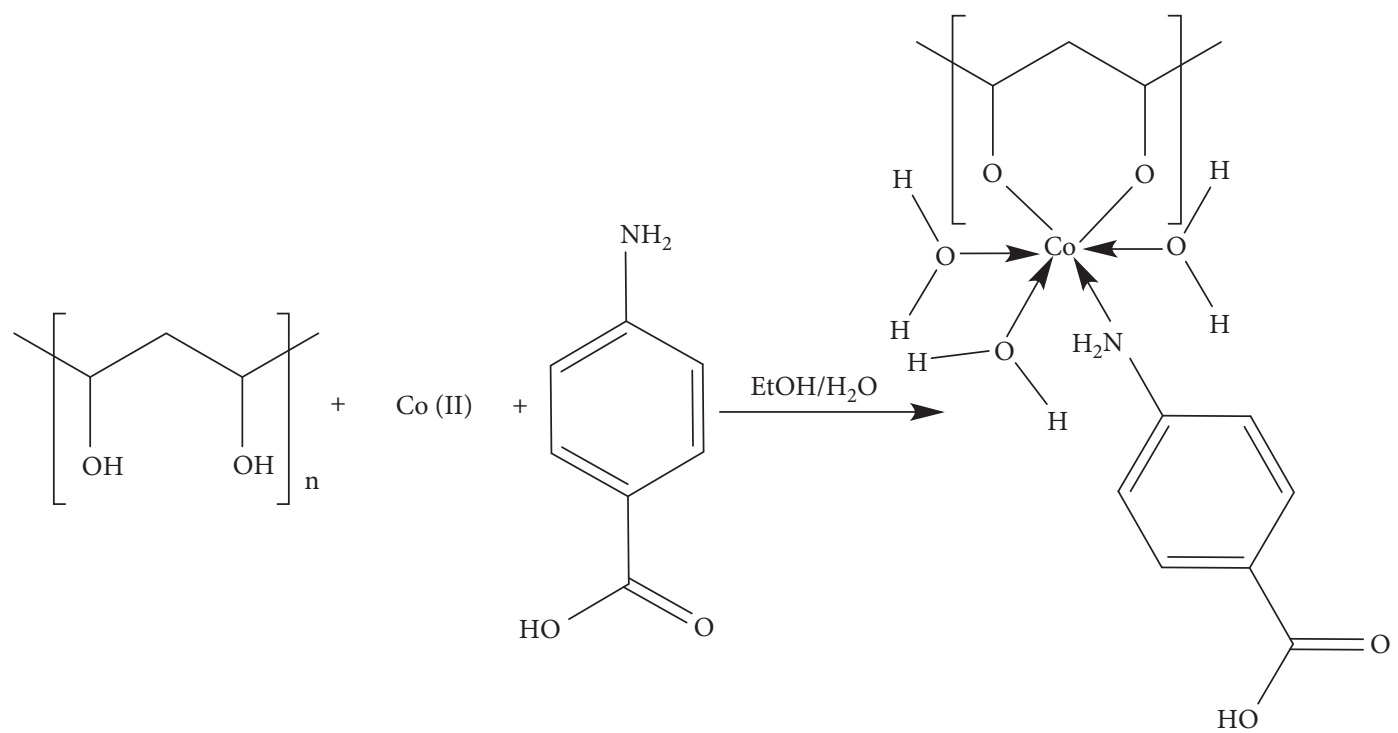

Scheme 1: Synthesis of the Co (II) Complex.

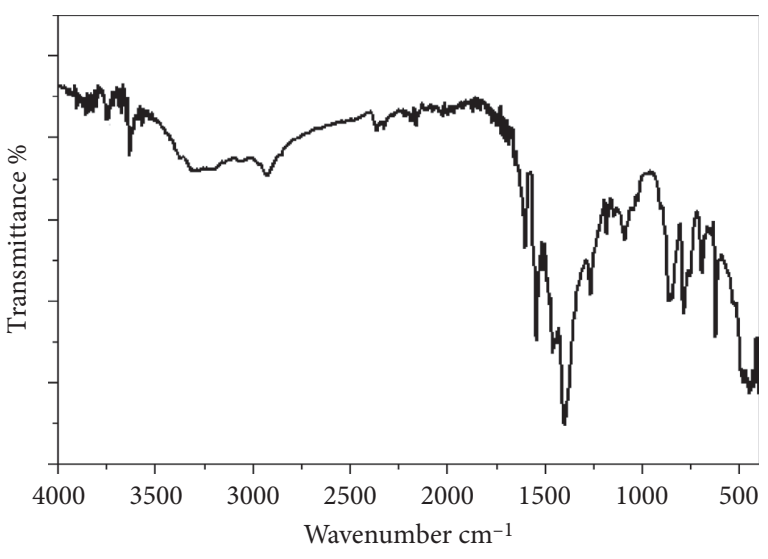

FIgURe 2: FT-IR of Co (II) complex.

steps: the Coats-Redfern and the Horowitz-Metzger [35, 36] methods (Figures 8 and 9). The kinetic parameters were calculated given to two equations and are listed in Table 1. The activation variables $\Delta \mathrm{H}^{*}, \Delta \mathrm{S}^{*}$, and $\Delta \mathrm{G}^{*}$ for the decomposition stages of this compound are recorded in Table 2. Negative $\Delta S^{*}$ numbers of the both steps of decomposition of the Co(II) complex propose that the stimulated compound is highly ordered than the reactants, and the reactions are lower than normal. The positive numbers of $\left(\Delta G^{*}\right)$ denote the decomposition reaction is not spontaneous.

Figure 10 illustrates the relation between $\alpha$ and temperature for the $\mathrm{Co}$ (II) compound in dynamic air. The figure indicates that the cobalt (II) compound is stable.

3.4. X-Ray Powder Diffraction (XRD), SEM, and TEM Analysis. XRD patterns of the CoO nanoparticles calcined at $550{ }^{\circ} \mathrm{C}$ are shown in Figure 11, which indicates cobalt oxide has a cubic phase structure (Table 3 ). The average grain size of $\mathrm{CoO}$ is determined using the Scherrer relation, and it was found to be around $54 \mathrm{~nm}$. Figure 12

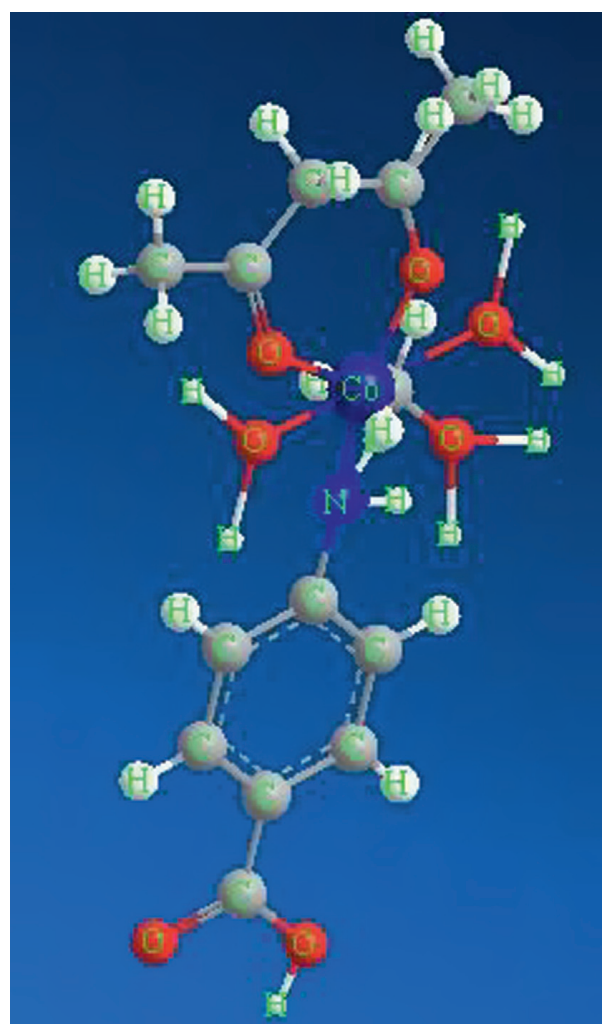

FIgURE 3: A perspective view of coordination round Co (II) ion.

shows the scanning electron microscopy image where the size of all mesoporous cobalt oxide nanocrystals is distorted growth to near-spherical. On the other hand (Figure 13), as shown in the TEM image, cobalt oxide nanoparticles (with a higher electron density) appear as darker spots. Moreover, the TEM picture shows clearly that the product is entirely composed of crystals with a relatively uniform, separate small spherical and cluster morphology. 


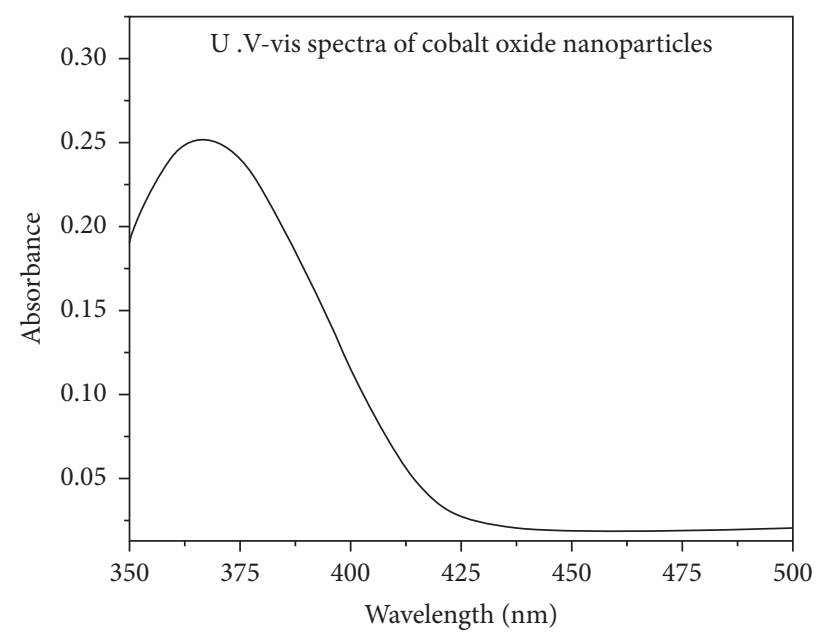

FIGURE 4: UV-visible image of cobalt (II) oxide nanoparticles.

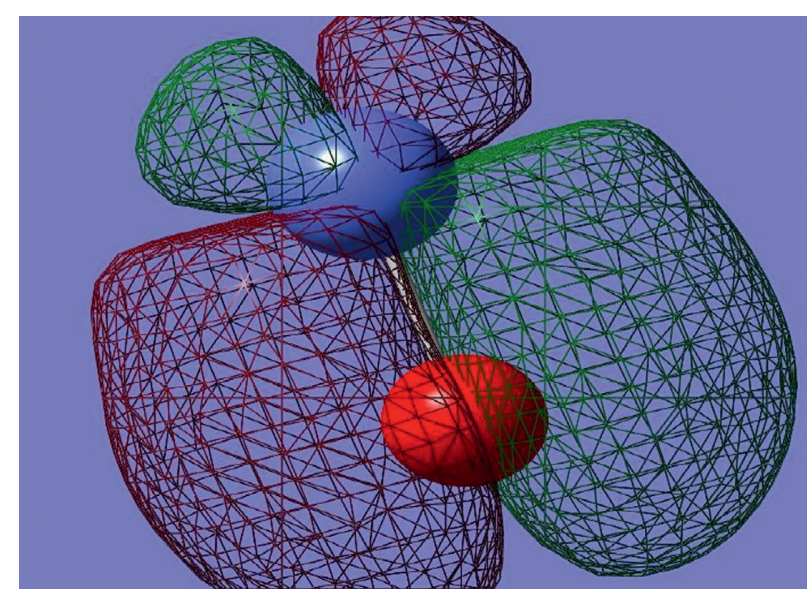

FIGURE 5: DFT molecular orbital plots for some frontier molecular orbitals of cobalt (II) oxide (HOMO and LUMO).

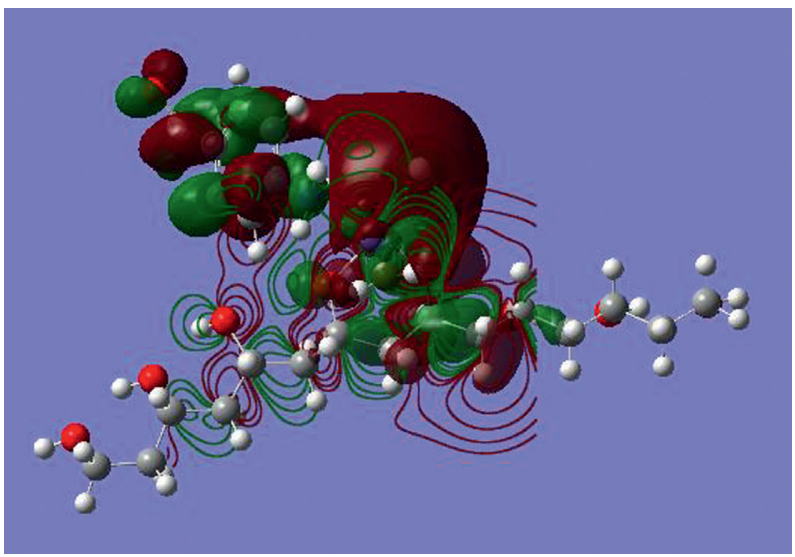

Figure 6: HOMO, LUMO (surfaces), and electron density (contours) for the Co (II) complex. 


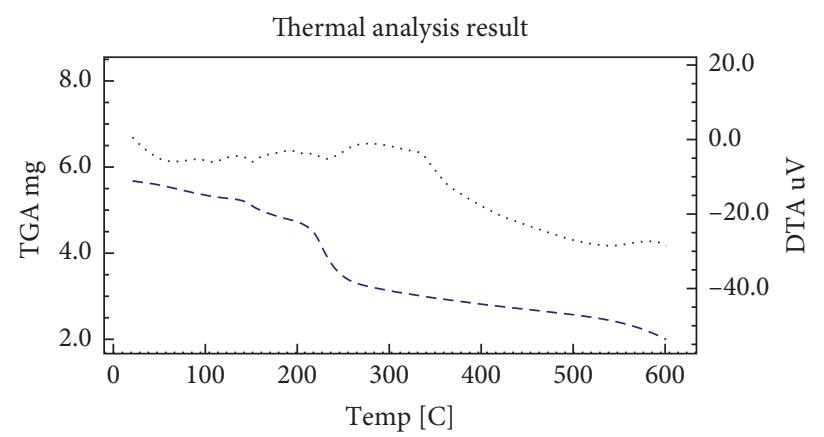

…. DTA

- - TGA

Figure 7: D.T.G and D.T.A curves of the Co (II) complex.

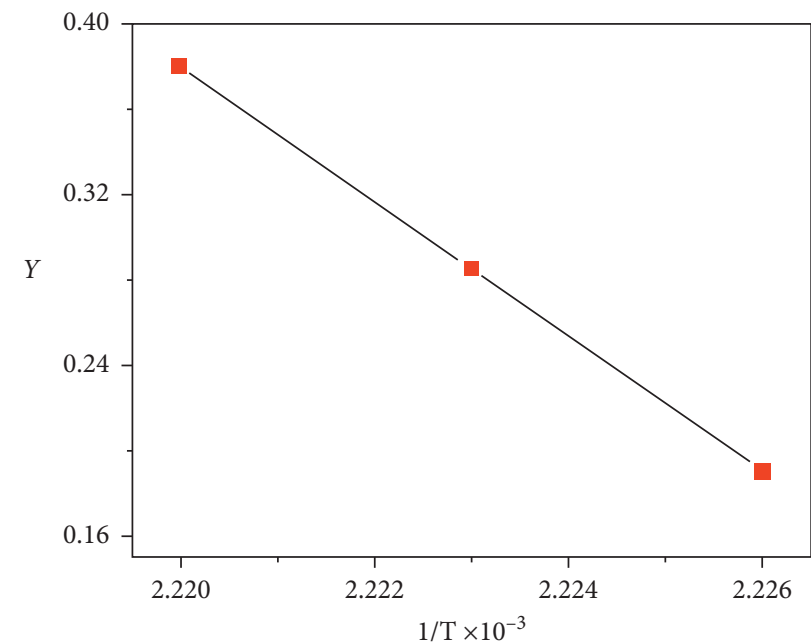

Figure 8: Coats-Redfern plots for the Co (II) complex.

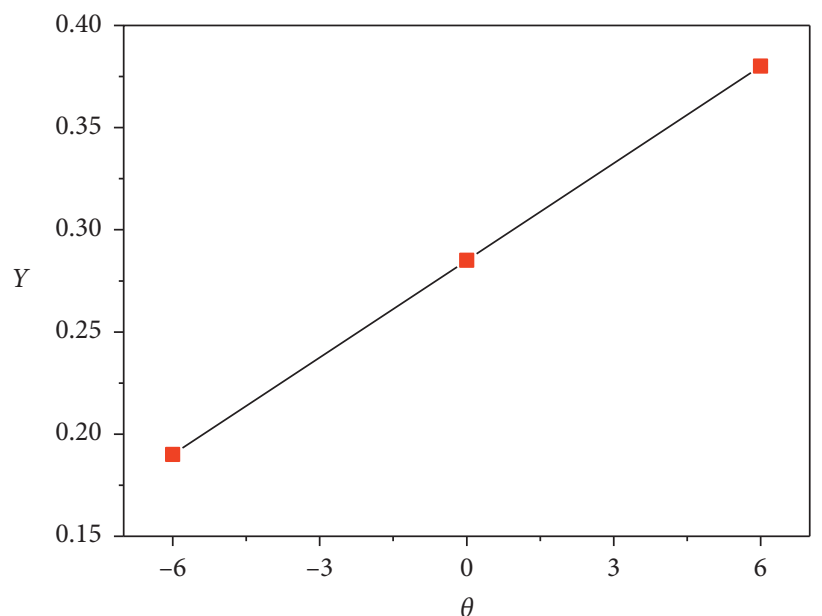

Figure 9: Horowitz-Metzger plots for the Co (II) complex. 
TABLE 1: Kinetic parameters for the thermal decomposition of the cobalt (II) complex.

\begin{tabular}{|c|c|c|c|c|c|c|c|c|}
\hline \multirow{2}{*}{ Step } & \multicolumn{4}{|c|}{ Coats-Redfern equation } & \multicolumn{4}{|c|}{ Horowitz-Metzger equation } \\
\hline & $n$ & $r$ & $E$ & $Z$ & $n$ & $r$ & $E$ & $Z \times 10^{2}$ \\
\hline \multirow{6}{*}{$2^{\text {nd }}$} & 0.00 & 0.9947 & 88.1 & 5.99 & 0.00 & 0.9994 & 93.6 & 3.51 \\
\hline & 0.33 & 0.9954 & 93.5 & 6.36 & 0.33 & 0.9958 & 101.0 & 7.56 \\
\hline & 0.50 & 0.9955 & 96.2 & 6.55 & 0.50 & 0.9962 & 103.8 & 7.60 \\
\hline & 0.66 & 0.9956 & 99.2 & 6.76 & 0.66 & 0.9965 & 106.8 & 7.64 \\
\hline & 1.00 & 0.9965 & 105.2 & 7.18 & 1.00 & 0.9970 & 112.7 & 7.72 \\
\hline & 2.00 & 0.9979 & 124.8 & 8.57 & 2.00 & 0.9982 & 132.2 & 7.97 \\
\hline
\end{tabular}

$\mathrm{E}$ in $\mathrm{KJ} \mathrm{mol}^{-1}$; underlined $r$ in all tables represents the best fit values of $n$ and $E$.

TABLE 2: Thermodynamic parameters for the thermal decomposition of the cobalt (II) complex.

\begin{tabular}{ccccc}
\hline Step & $n$ & $\Delta S^{*}$ & $\Delta H^{*}$ & $\Delta G^{*}$ \\
\hline & 0.00 & -203.51 & 89.97 & 180.93 \\
& 0.33 & -197.13 & 97.32 & 185.43 \\
$2^{\text {nd }}$ & 0.50 & -197.09 & 100.13 & 188.22 \\
& 0.66 & -197.05 & 103.10 & 191.18 \\
& 1.00 & -196.96 & 109.06 & 197.10 \\
& 2.00 & -196.70 & 128.57 & 216.49 \\
\hline
\end{tabular}

$\Delta \mathrm{H}^{*}$ and $\Delta \mathrm{G}^{*}$ in $\mathrm{kJ} \mathrm{mol}{ }^{-1}$ and $\Delta \mathrm{S}^{*}$ in $\mathrm{kJ} \mathrm{mol}^{-1} \mathrm{~K}^{-1}$.

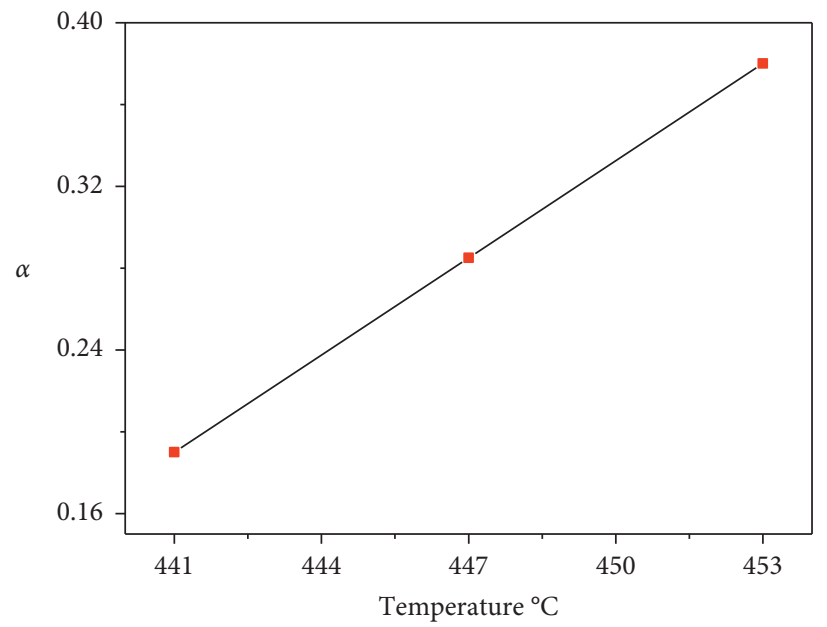

Figure 10: Fraction decomposed $(\alpha)$ and temperature plots of the Co (II) complex.

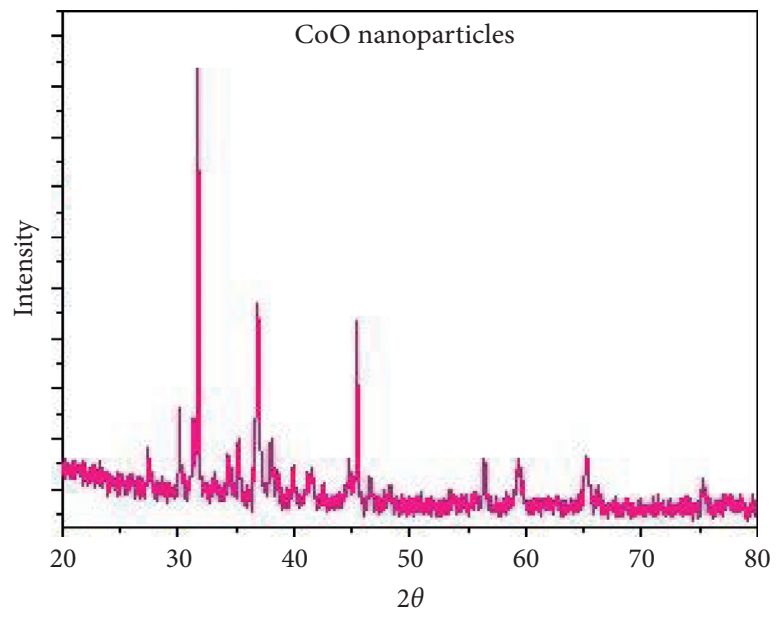

FIGURE 11: XRD of cobalt oxide nanoparticles. 
TABle 3: XRD crystal data of the CoO NPs.

\begin{tabular}{lc}
\hline Parameters & CoO \\
\hline Empirical formula & CoO \\
Formula weight & 74.92 \\
Crystal system & Cubic \\
a $(\AA)$ & 8.08 \\
b $(\AA)$ & 8.08 \\
$\mathrm{c}(\AA)$ & 8.08 \\
$\alpha\left(^{\circ}\right)$ & 90.00 \\
$\beta\left({ }^{\circ}\right)$ & 90.00 \\
$\gamma\left({ }^{\circ}\right)$ & 90.00 \\
Volume of the unit cell $(\AA 3)$ & 528.12 \\
Particle size $(\mathrm{nm})$ & 54 \\
\hline
\end{tabular}

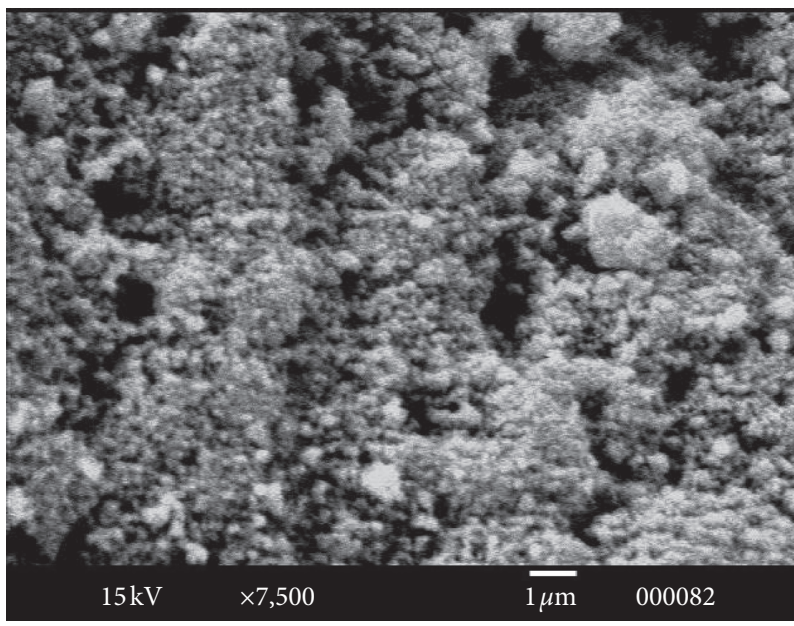

FIGURE 12: Scanning electron microscope image of cobalt oxide nanoparticles.

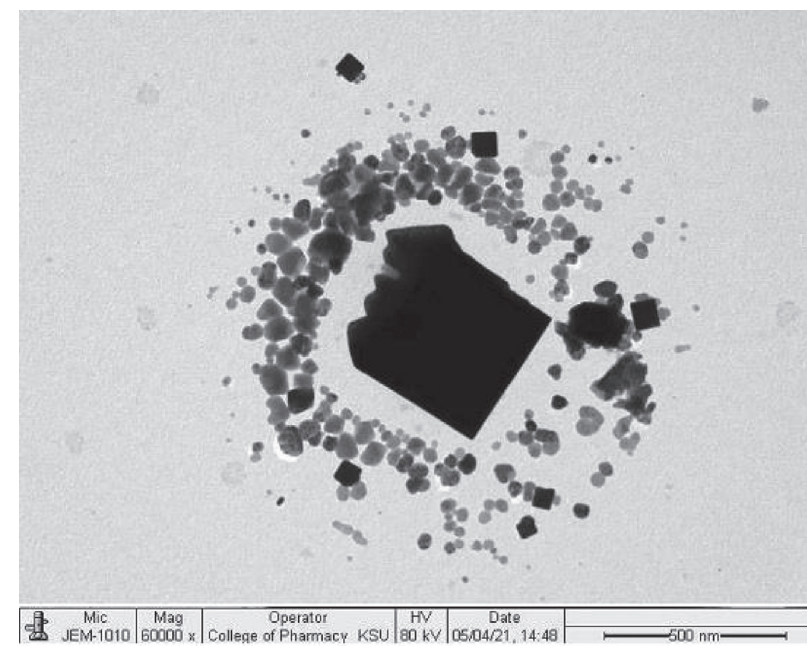

FIgURE 13: Transmission electron microscope image of cobalt oxide nanoparticles. 


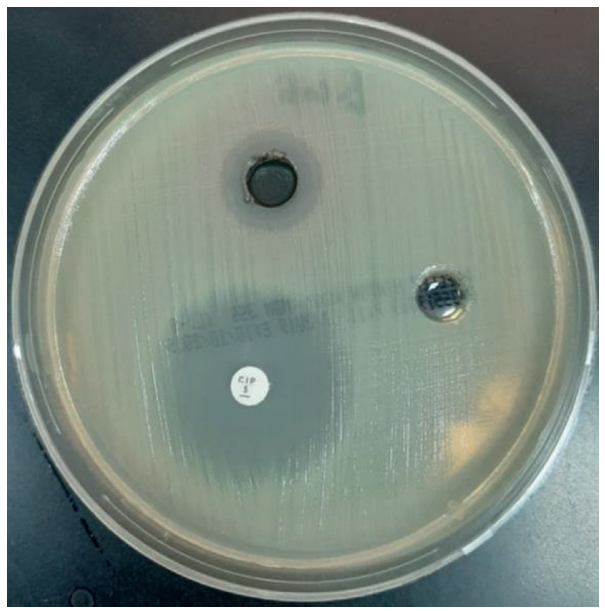

FIGURE 14: Microbiological screening of the CoO NPs against E. coli.

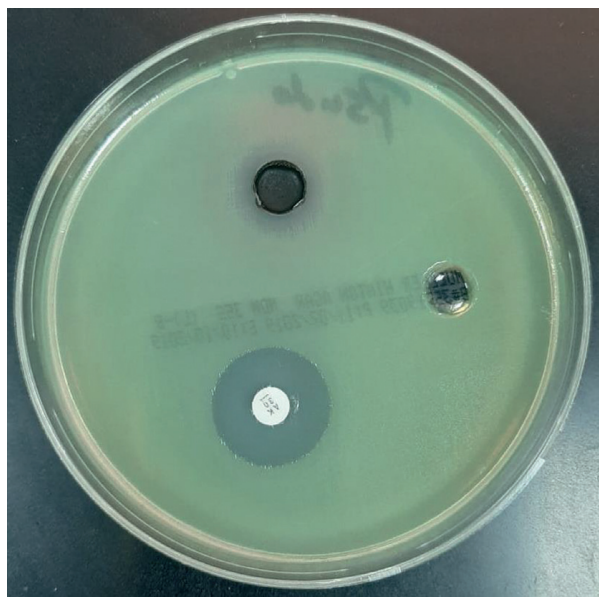

Figure 15: Microbiological screening of the CoO NPs against Pseudomonas aeruginosa.

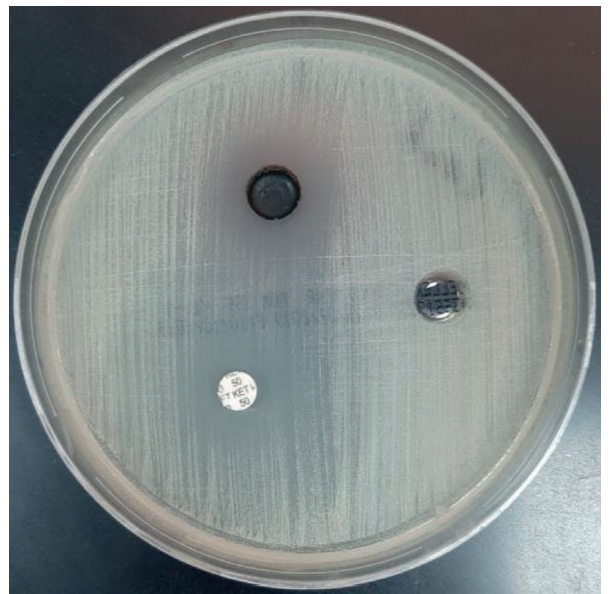

Figure 16: Microbiological screening of the CoO NPs against Candida albicans. 
3.5. Antimicrobial Activity of Cobalt Oxide Nanoparticles. Cobalt oxide nanoparticles are well known to have an antimicrobial activity. In this study, it was clear that cobalt oxide nanoparticles had good antimicrobial activity against Gram-negative bacilli and yeast such as fungi, but no activity against Gram-positive cocci. The inhibition zones $(\mathrm{mm})$ for these test organisms are as follows: Escherichia coli $16 \mathrm{~mm}$, Pseudomonas aeruginosa $15 \mathrm{~mm}$, and Candida albicans $20 \mathrm{~mm}$. The big hole is for cobalt (II) oxide nanoparticle, and the others are for the antimicrobial disk and solvent. The microbiological screening of the $\mathrm{CoO}$ NPs is shown in Figures 14-16.

\section{Conclusions}

In conclusion, we have demonstrated the facile synthesis of the spherical formed aggregates of cubic-CoO (NPs) by the calcination method of the Co (II) complex. The phase and morphology of the cobalt (II) oxide nanocrystals are characterised by XRD, SEM, and TEM. Results of X-ray sample display that all peaks can be well listed to the phase of CoO. SEM micrographs offer that there are many micropores through the nanocrystals for the sample calcined at $550^{\circ} \mathrm{C}$ for 3 hours. Also, the antimicrobial activity is tested against standard fungal and bacterial species, and the results present that the calcination method increased antibacterial and antifungal activity than other preparation methods due to small particle size, production of reactive oxygen species (ROS), and large surface area.

\section{Data Availability}

The data used to support the findings of this study are available within the article.

\section{Conflicts of Interest}

The authors declare that they have no conflicts of interest.

\section{References}

[1] J. A. Rodríguez and M. Fernández-Garćia, Synthesis, Properties and Applications of Oxide Nanoparticles, Wiley, Hoboken, NJ, USA, 2007.

[2] R. W. G. Wyckoff, Crystal Structures, Wiley, New York, NY, USA, 2nd edition, 1964.

[3] M. L. Trudeau and J. Y. Ying, "Nanocrystalline materials in catalysis and electrocatalysis: structure tailoring and surface reactivity," Nanostructured Materials, vol. 7, no. 1-2, pp. 245-258, 1996.

[4] P. Ayyub, V. R. Palkar, S. Chattopadhyay, and M. Multani, "Size-induced diffuse phase transition in the nanocrystalline ferroelectric $\mathrm{PbTiO}_{3}$," Pysical Review B, vol. 52, no. 18, pp. 13177-13183, 1995.

[5] J. Schoiswohl, M. Sock, S. Eck et al., "Atomic-level growth study of vanadium oxide nanostructures on Rh (III)," Physical Review B, vol. 69, no. 15, 2004.

[6] T. Merciris, F. Valensi, and A. Hamdan, "Synthesis of nickel and cobalt oxide nanoparticles by pulsed underwater spark discharges," Journal of Applied Physics, vol. 129, no. 6, 2021.
[7] M. T. Makhlouf, B. M. Abu-Zied, and T. H. Mansoure, "Effect of calcination temperature on the $\mathrm{H}_{2} \mathrm{O}_{2}$ decomposition activity of nano-crystalline $\mathrm{Co}_{3} \mathrm{O}_{4}$ prepared by combustion method," Applied Surface Science, vol. 274, pp. 45-52, 2013.

[8] M. Ibrahim and S. El-Molla, "Physicochemical, catalytic and antimicrobial activities of porous cobalt oxide nanoparticles (kinetics study of $\mathrm{H}_{2} \mathrm{O}_{2}$ decomposition using fluorometric and gasometric methods)," Canadian Journal of Chemistry, vol. 96, no. 8), 2018.

[9] M. S. Samuel, E. Selvarajan, T. Mathimani et al., "Green synthesis of cobalt-oxide nanoparticle using jumbo muscadine (Vitis Rotundifolia): characterization and photo-catalytic activity of acid blue-74," Journal of Photochemistry and Photobiology B: Biology, vol. 211, Article ID 112011, 2020.

[10] J. Ryu, S.-W. Kim, K. Kang, and C. B. Park, "Synthesis of diphenylalanine/cobalt oxide hybrid nanowires and their application to energy storage," ACS Nano, vol. 4, no. 1, pp. 159-164, 2009.

[11] H. Sun, H. Liang, G. Zhou, and S. Wang, "Supported cobalt catalysts by one-pot aqueous combustion synthesis for catalytic phenol degradation," Journal of Colloid and Interface Science, vol. 394, pp. 394-400, 2013.

[12] S. Stankic, S. Suman, F. Haque, and J. Vidic, "Pure and multi metal oxide nanoparticles: synthesis, antibacterial and cytotoxic properties," Journal of Nanobiotechnology, vol. 14, p. 73, 2016.

[13] S. A. El-Molla and H. R. Mahmoud, "Synthesis, textural and catalytic properties of nanosized $\mathrm{Fe}_{2} \mathrm{O}_{3} / \mathrm{MgO}$ system," Materials Research Bulletin, vol. 48, no. 10, pp. 4105-4111, 2013.

[14] K. P. Shinde, S. S. Pawar, P. M. Shirage, and S. H. Pawar, "Studies on morphological and magnetic properties of $\mathrm{La}_{1-\mathrm{x}} \mathrm{Sr}_{\mathrm{x}} \mathrm{MnO}_{3}$," Applied Surface Science, vol. 258, no. 19, pp. 7417-7420, 2012.

[15] D. Xu, Y. Bu, W. Tan, and Q. Zhong, "Structure and redox properties of perovskite $\left.\mathrm{Y}_{0.9} \mathrm{Sr}_{0.1} \mathrm{Cr}_{1-\mathrm{x}} \mathrm{Fe}_{\mathrm{x}} \mathrm{O}_{3-\delta}\right)$," Applied Surface Science, vol. 268, pp. 246-251, 2013.

[16] M. Saif, S. Aboul-Fotouh, S. El-Molla, M. Ibrahim, and L. Ismail, "Improvement of the structural, morphology, and optical properties of $\mathrm{TiO}_{2}$ for solar treatment of industrial wastewater," Journal of Nanoparticle Research, Springer, Cham, Switzerland, 2012.

[17] N. Venugopal, G. Krishnamurthy, H. Bhojyanaik, M. Madhukar Naik, and N. Sunilkumar, "Synthesis, characterization, and biological activity of $\mathrm{Cu}$ (II) and Co (II) complexes of novel N1, N 2 -bis (4- methyl quinolin-2-yl) benzene-1,2-diamine: $\mathrm{CuO}$ and $\mathrm{CoO}$ nanoparticles derived from their metal complexes for photocatalytic activity," Inorganic and Nano-Metal Chemistry, vol. 30, no. 9, pp. 1-10, 2020.

[18] R. Bhuvaneswari, S. Karthikeyan, S. Selvasekarapandian et al., "Preparation and characterization of PVA complexed with amino acid, proline," Ionics, vol. 21, no. 2, pp. 387-399, 2015.

[19] I. O. Ali, H. S. Nassar, K. S. El-nasser, A. Bougarech, M. Abid, and A. A. Elhenawy, "Synthesis and characterization of $\mathrm{Mn}^{\mathrm{II}}$ and $\mathrm{Co}^{\mathrm{II}}$ complexes with poly (vinyl alcoholnicotinic acid) for photocatalytic degradation of indigo carmine dye," Inorganic Chemistry Communications, vol. 124, Article ID 108360, 2021.

[20] A. Sinha, S. K. Das, V. Rao, and P. Ramachandrarao, "Patterning of copper particles on polymeric surface," Journal of Materials Research, vol. 16, no. 5, pp. 1354-1357, 2011.

[21] W. Hosny and P. Khalaf-Alaa, "Potentiometric study and biological activity of Some metal ion complexes of polyvinyl 
alcohol (PVA)," International Journal of Electrochemical Science, vol. 8, pp. 1520-1533, 2013.

[22] M. Al-Fakeh and F. Alminderej, "New method for the preparation and biological activity of $\mathrm{CuO}$ nanoparticles from a mixed PVA and 2-Aminobenzothiazole complex," International Journal of Chem Tech Research, vol. 11, pp. 442-449, 2018.

[23] P. Saokham, K. Burapapadh, P. Praphanwittaya, and T. Loftsson, "Characterization and evaluation of ternary complexes of Ascorbic acid with $\gamma$-cyclodextrin and poly (vinyl alcohol)," International Journal of Molecular Sciences, vol. 21, no. 12, p. 4399, 2020.

[24] M. Brza, S. B. Aziz, H. Anuar, F. Ali, and E. Dannoun, "Tea from the drinking to the synthesis of metal complexes and fabrication of PVA based polymer composites with controlled optical band gap," Scientific Reports, vol. 10, 2020.

[25] X.-X. Cheng, S. Hojaghani, M.-L. Hu, M. Hosaini Sadr, and A. Morsali, "Sonochemical synthesis and characterization of new nanostructures cobalt (II) metal-organic complexes derived from the azo-coupling reaction of 4 -amino benzoic acid with anthranilic acid, salicylaldehyde and 2-naphtol," Ultrasonics Sonochemistry, vol. 37, pp. 614-622, 2017.

[26] J. Kalembkiewicz, M. Kosińska, and M. Zapała, "Complexes of aminobenzoic acids: a comprehensive review concerning synthesis, physical chemistry, structure and application," Coordination Chemistry Reviews, vol. 348, pp. 25-53, 2017.

[27] ADF2.3 and ADF2002.01; SCM: Theoretical Chemistry, Vrije Universiteit, Amsterdam, The Netherlands,http://www.scm. com.

[28] H. S. Mansur, R. L. Oréfice, and A. A. P. Mansur, "Characterization of poly (vinyl alcohol)/poly (ethylene glycol) hydrogels and PVA-derived hybrids by small-angle X-ray scattering and FTIR spectroscopy," Polymer, vol. 45, no. 21, pp. 7193-7202, 2004.

[29] M. S. Al-Fakeh, "Synthesis, thermal stability and kinetic studies of copper (II) and cobalt (II) complexes derived from 4-aminobenzohydrazide and 2-mercaptobenzothiazole," European Chemical Bulletin, vol. 9, no. 12, pp. 403-409, 2020.

[30] A. Lisdiana, H. Wahab, S. B. Rahardjo, and T. E. Saraswati, "Synthesis and characterization of iron (II)-4- aminobenzoic acid complex as potent antibacterial agent," International Journal of Chem Tech Research, vol. 14, no. 1, pp. 42-49, 2021.

[31] M. S. Al-Fakeh, "Synthesis and characterization of coordination polymers of 1,3-di (4-pyridyl)-propane and 2Aminobenzothiazole with Mn (II), Co (II), Cu (II) and Ni (II) ions," Journal of Chemical and Pharmaceutical Research, vol. 10, no. 2, pp. 77-83, 2018.

[32] K.-L. Liang, Y.-C. Wang, W.-L. Lin, and J.-J. Lin, "Polymerassisted self-assembly of silver nanoparticles into interconnected morphology and enhanced surface electric conductivity," RSC Advances, vol. 4, no. 29, p. 15098, 2014.

[33] K. S. Ambili and J. Thomas, "Synthesis of hybrid materials by immobilizing para-aminobenzoic acid complexes of Eu3+ and $\mathrm{Tb} 3+$ in zeolite $\mathrm{Y}$ and their luminescent properties," Journal of Porous Materials, vol. 27, no. 3, pp. 755-764, 2020.

[34] A. Aly, M. Ghandour, B. Abu-Zied, and M. Al-Fakeh, "Environmentally Relevant supramolecular metal coordination polymers derived from 5-(3-pyridyl)- 1,3,4-Oxadiazole2-thiole and imidazole," Oriental Journal of Chemistry, vol. 29, pp. 149-159, 2013.

[35] A. Coats and J. Redfern, "Kinetic parameters from thermogravimetric data," Nature, vol. 201, pp. 68-69, 1964.
[36] H. H. Horowitz and G. Metzger, "A new analysis of thermogravimetric traces," Analytical Chemistry, vol. 35, no. 10, pp. 1464-1468, 1963. 